\title{
Benefits, pitfalls, and future design of population-based registers in neurodegenerative disease
}

James P.K. Rooney, MSc Carol Brayne, $\mathrm{PhD}$

Katy Tobin, $\mathrm{PhD}$

Giancarlo Logroscino,

$\mathrm{PhD}$

M. Maria Glymour, ScD

Orla Hardiman, MD

Correspondence to

Dr. Rooney:

jrooney@rcsi.ie
Supplemental data at Neurology.org

\section{ABSTRACT}

Population-based disease registers identify and characterize all cases of disease, including those that might otherwise be neglected. Prospective population-based registers in neurodegeneration are necessary to provide comprehensive data on the whole phenotypic spectrum and can guide planning of health services. With the exception of the rare disease amyotrophic lateral sclerosis, few complete population-based registers exist for neurodegenerative conditions. Incomplete ascertainment, limitations and uncertainty in diagnostic categorization, and failure to recognize sources of bias reduce the accuracy and usefulness of many registers. Common biases include population stratification, the use of prevalent rather than incident cases in earlier years, changes in disease understanding and diagnostic criteria, and changing demographics over time. Future registers are at risk of funding shortfalls and changes to privacy legislation. Notwithstanding, as heterogeneities of clinical phenotype and disease pathogenesis are increasingly recognized in the neurodegenerations, well-designed longitudinal population-based disease registers will be an essential requirement to complete clinical understanding of neurodegenerative diseases. Neurology ${ }^{\circledR} 2017 ; 88: 1-9$

\section{GLOSSARY}

$\mathbf{A D}=$ Alzheimer disease; $\mathbf{A L S}=$ amyotrophic lateral sclerosis; $\mathbf{E H R}=$ electronic health record; $\mathbf{F T D}=$ frontotemporal dementia; PD = Parkinson disease.

The extent of heterogeneity in clinical phenotype and disease pathogenesis is increasingly recognized in the neurodegenerations, and population-based disease registers can provide highquality longitudinal data that enable comparative analysis of demographics, phenotype, and outcome across different geographic regions.

Registers differ from other types of patient-related data such as electronic health records (EHRs), routine statistics held within national health services, and surveillance based on representative samples (e.g., the US National Health and Nutrition Examination Survey). Register data are actively collected (core data ${ }^{1,2}$ ), and require rigorous standardized management. By contrast, EHRs are collected at the time of patient encounter and are coded based on the diagnosis at that time. While such records can be useful as source datasets in the construction of registers, EHRs alone are rarely sufficiently accurate or complete to enable the detailed surveillance associated with disease registers, as exemplified recently by comparison of an administrative database with a population-based register for amyotrophic lateral sclerosis (ALS) in France. ${ }^{3}$

Restricted quality registers for neurodegeneration have been established in some regions, such as SveDem, an Internet-based quality registry that includes patients from memory clinics and primary care units with an estimated capture rate of 36\%. ${ }^{4}$ Similarly, a French Dementia Register comprises $84 \%$ of memory clinics. ${ }^{5}$ These registers are not designed to recruit within the entire population, and while valuable in defining the characteristics of those enrolled, they

From the Academic Unit of Neurology (J.P.K.R., K.T., O.H.), Trinity Biomedical Sciences Institute, Trinity College Dublin, Ireland; Department of Public Health and Primary Care (C.B.), School of Clinical Medicine, University of Cambridge, UK; Department of Basic Medical Sciences, Neuroscience and Sense Organs (G.L.), University of Bari “Aldo Moro,” Bari; Unit of Neurodegenerative Diseases (G.L.), Department of Clinical Research in Neurology, University of Bari “Aldo Moro," Pia Fondazione Cardinale G. Panico, Tricase, Lecce, Italy; Department of Epidemiology and Biostatistics (M.M.G.), University of California, San Francisco; and Department of Neurology (O.H.), Beaumont Hospital, Dublin, Ireland. Go to Neurology.org for full disclosures. Funding information and disclosures deemed relevant by the authors, if any, are provided at the end of the article. 
are by definition limited by their design and cannot provide an entire perspective of all cases with dementia.

Most population-based registers require a number of years of activity to ensure that early biases are resolved. Once fully functional, they can facilitate detailed analyses of disease heterogeneity, risk, health planning, and monitoring of disease burden in the population. ${ }^{6-14}$

The purpose of this review is to analyze the methods underpinning existing populationbased registers for neurodegeneration, to provide insights into hidden biases that can confound analysis of register-based data, and to suggest methods to minimize errors of interpretation.

EXISTING POPULATION-BASED REGISTERS IN NEURODEGENERATION Few true populationbased registers of adult neurodegenerative disease currently exist. Examples of successful registers include those developed for $\mathrm{ALS}^{6-8}$ and frontotemporal dementia (FTD). ${ }^{9-11}$ The European ALS registers can be viewed as gold standards for rare neurodegenerative diseases, as they have provided important insights into disease pathogenesis, clinical heterogeneity, and spatial distribution. ${ }^{6,12-14}$

Conversely, a relatively low number of true population-based registers for commoner conditions such as Parkinson disease (PD) and Alzheimer disease (AD) have been established, primarily in Scandinavia, the Netherlands, and some regions of the United States. ${ }^{9,15,16}$ The Nebraska Parkinson's Disease Register $^{17}$ has $98 \%$ ascertainment and uses capturerecapture analysis, ${ }^{15,18,19}$ which combines information from multiple ascertainment sources to estimate the number of cases that are likely to have been missed. Logistics of collection are aided through the support of state law, and the register ascertains from multiple sources with a verification process for all reported cases. ${ }^{15,17}$ This register is feasible in part due to the low population of Nebraska (1.8 million), which allows identification of all cases in a relatively laborintensive manner. Given the high prevalence of PD in most countries (329.3/100,000 in Nebraska $\left.{ }^{15}\right)$, the costs of directly applying these methods in more populous regions would be considerable.

As demonstrated by the Nebraska register, registers require active ascertainment from multiple sources to exclude possible coding errors, and to identify those within the population who may not engage with specialist services (figure). Exclusive reliance on passive capture through existing records risks ascertainment bias. For example, an approach for $\mathrm{PD}$ in Thailand (population 67 million) using 3 independent administrative sources achieved only $66 \%$ case ascertainment. ${ }^{20}$

Registers from Scandinavia, ${ }^{21}$ Canada, ${ }^{22}$ the United States, ${ }^{23}$ and Taiwan ${ }^{24}$ have ascertained cases using administrative EHRs. As exemplified by a prescription-based register for $\mathrm{AD}$ in Finland requiring stringent proof of diagnosis, such an approach can successfully exclude coding errors if administrative records are a priori designed to ensure reliability in ascertainment. However, use of EHRs that focus exclusively on drug prescription may also introduce other types of selection biases, as it is unlikely that all patients with neurodegenerative disease will use targeted medications. ${ }^{25}$ Even in countries with welldeveloped social health systems such as Finland, additional sources of ascertainment are required.

In general, studies that have ascertained cases only from specialist clinics, such as behavioral or old age psychiatry clinics for $\mathrm{AD}$, are also likely to be biased, ${ }^{26}$ as referral patterns can vary based on age, income levels, and educational status (figure). There is evidence that specialist clinics are likely to exclude very early and very advanced cases, ${ }^{26-29}$ and those with a very aggressive course, as exemplified by data from the Irish ALS Register (table 1). Extrapolation from these types of datasets is limited by selective participation and attrition, particularly in dementia studies. As cohorts are more likely to be younger, interpretation of datasets from specialist memory clinics cannot be generalizable to the entirety of people with dementia, the majority of whom within the population are over 85 years of age. ${ }^{30}$ Because of this, biomarkers and prognostic indicators that are inferred from analysis of cohorts cannot be applied to the community at large. This high likelihood of a false-positive effect has been shown for mild cognitive impairment. ${ }^{29}$

\section{ALTERNATIVE APPROACHES TO POPULATION- BASED REGISTERS: POPULATION-BASED} COHORT STUDIES Given the known logistical challenges associated with full ascertainment of patients with common neurodegenerative disease, other methods based on complete follow-up of an entire or a randomly selected population in a small area have been used. All residents within a specific age range are regularly screened for the disease and exposures of interest, and are followed up regardless of the presence or absence of diagnosis. Examples of these types of studies include the Framingham Heart Study, ${ }^{31}$ the Swedish National Study on Aging and Care, ${ }^{32}$ and the Rotterdam Study in Holland. As exemplified by the Rotterdam study, ${ }^{33-35}$ all residents within a specific region and age category (over 55) are invited to participate in a longitudinal study of multiple health outcomes. These types of studies repeatedly evaluate the participating subject cohorts over many years, and 


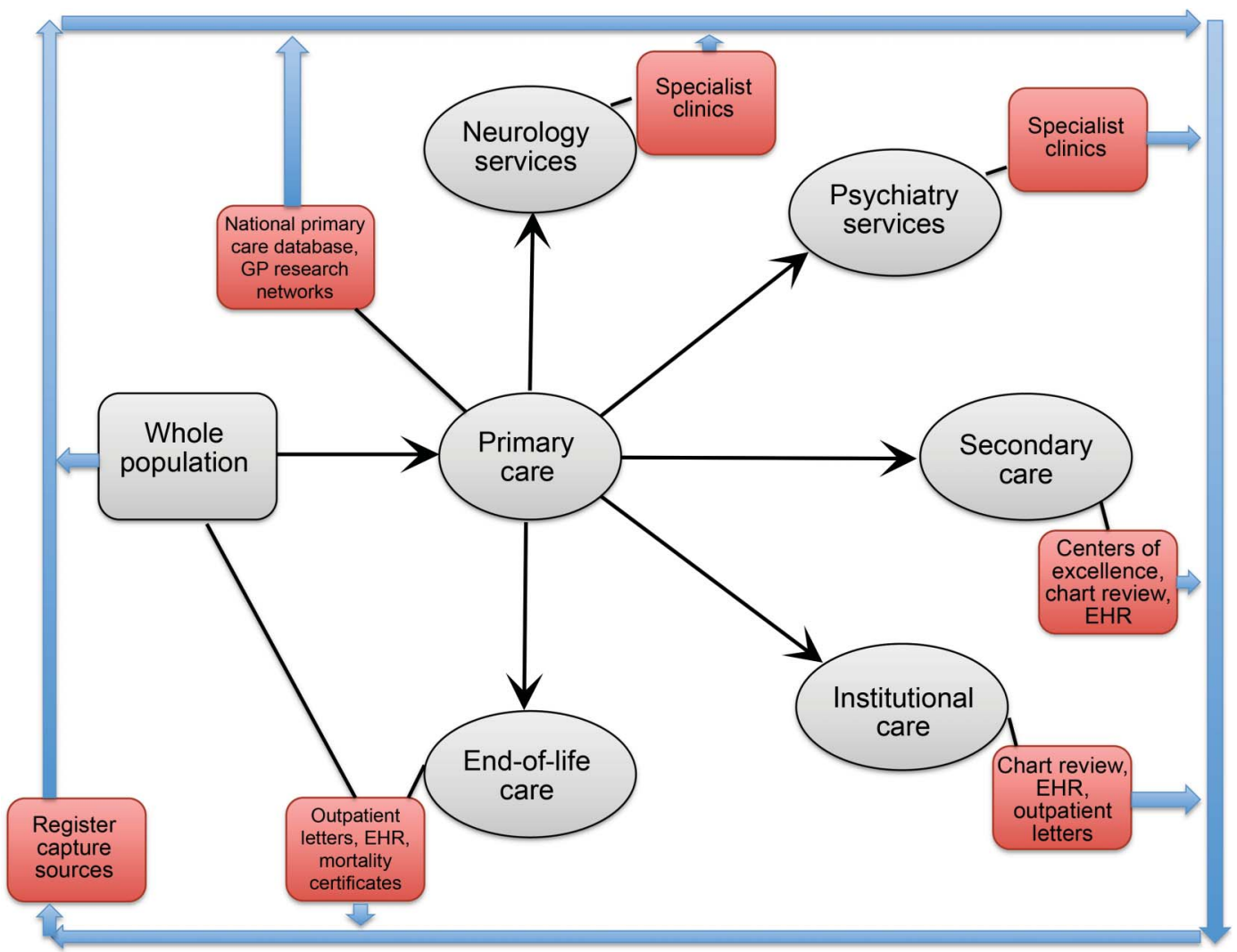

Population-based registers must aim to reliably and consistently identify patients from all pathways, requiring government and institutional support, sufficient funding and manpower, and local expertise. Social, demographic, and clinical selection biases can influence the characteristics of patients following each pathway, and this can vary by disease and by health care system. As a result, research studies recruiting from single pathways will inherit these biases. Functioning population-based registers overcome these biases via inclusion of all pathways. Adapted from The Lancet (Brayne C, Davis D. Making Alzheimer's and dementia research fit for populations. Lancet 2012;380:1441-1443), @ 2012, with permission from Elsevier. ${ }^{26}$

statistical assessment of associations between risk factors and disease incidence is determined. A major strength is that members of the population are regularly examined, including those who have not fully engaged with health services. New cases are identified at each repeated screening/visit that occurs within the target region. This differs from the traditional concept of a disease register, as the parent study is usually not restricted to a single diagnostic criterion for inclusion, but instead typically includes a range of diseases across specialties. ${ }^{33}$ However, by capturing information about diverse diseases within and across specialties, this method can be of great utility in collecting a true population-based estimate of common but heterogeneous conditions such as dementia or parkinsonism. ${ }^{33}$ It also has the advantage that it can capture ongoing changes in diagnostic criteria and can determine whether revised diagnoses predict outcomes. Another advantage of this approach is that the inclusion of healthy people within the cohort allows the rigorous assessment of both premorbid exposures and symptoms. ${ }^{33,35}$ This is valuable because the pathogenesis of some forms of neurodegeneration (e.g., dementia and PD) is known to be multifactorial, and utilization of a design that continuously samples and reassesses a population regardless of their diagnosis can facilitate large longitudinal studies that provide more accurate data pertaining to both clinical phenotype and risk. This approach has a further advantage of enabling recognition and evaluation of clinical conditions (comorbidities) and other changes that typically occur in elderly populations.

However, methods that depend on regular assessment of specified populations also have important limitations. They are not feasible for rare neurodegenerative conditions as it would be impossible to sample a sufficiently large population to enable detection of a large number of cases. Moreover, all cohorts are vulnerable to selective enrollment and attrition. For example, cognitive impairment strongly predicts study dropout, ${ }^{36,37}$ potentially leading to substantial underestimates of dementia incidence. Extremely fast-progressing cases may never be identified in cohorts, because a patient may be diagnosed and die during the interval between 2 assessment waves. This can result in misleading estimates of disease 


\begin{tabular}{|c|c|c|c|c|}
\hline \multirow[t]{2}{*}{ Table 1} & \multirow[b]{2}{*}{$\begin{array}{l}\text { Population sample } \\
(\mathrm{n}=1,282)\end{array}$} & \multirow[b]{2}{*}{$\begin{array}{l}\text { Clinic attenders } \\
\text { only }(n=814)\end{array}$} & \multirow[b]{2}{*}{$\begin{array}{l}\text { Clinic nonattenders } \\
\text { only }(n=468)\end{array}$} & \multirow[b]{2}{*}{$\begin{array}{l}p \text { Value (clinic attenders } \\
\text { vs nonattenders) }\end{array}$} \\
\hline & & & & \\
\hline \multicolumn{5}{|l|}{ Sex, n (\%) } \\
\hline Male & $723(56.4)$ & $458(56.3)$ & 265 (56.5) & \\
\hline Female & 559 (43.6) & $356(43.7)$ & $203(43.4)$ & 0.907 \\
\hline Mean (SD) age at onset, y & $64.6(11.9)$ & $62.3(11.7)$ & $68.4(11.5)$ & $<0.0001^{\mathrm{a}, \mathrm{b}}$ \\
\hline \multicolumn{5}{|l|}{ Diagnostic delay, wk, n (\%) } \\
\hline$<31$ & $380(33.8)$ & 232 (31.9) & $148(37.4)$ & \\
\hline $31-55$ & 371 (33.0) & 240 (33.0) & 131 (33.1) & \\
\hline$>55$ & 372 (33.2) & 255 (35.1) & $117(29.6)$ & 0.098 \\
\hline \multicolumn{5}{|l|}{ Site of onset, $n(\%)$} \\
\hline Limb & $743(58.4)$ & $516(63.8)$ & 227 (48.7) & \\
\hline Bulbar & 465 (36.5) & 257 (31.8) & $208(44.6)$ & \\
\hline Limb and bulbar & $65(5.1)$ & $34(4.2)$ & $31(6.7)$ & $<0.001^{\mathrm{b}}$ \\
\hline \multicolumn{5}{|l|}{ El Escorial, n (\%) } \\
\hline Definite & 709 (56.2) & $436(54.1)$ & $273(59.7)$ & \\
\hline Probable & 386 (30.6) & 255 (31.7) & $131(28.7)$ & \\
\hline Possible & $153(12.1)$ & 107 (13.3) & $46(10.1)$ & \\
\hline Suspected & $14(1.1)$ & $7(0.9)$ & 7 (1.5) & 0.101 \\
\hline $\begin{array}{l}\text { Median unadjusted survival } \\
(95 \% \mathrm{CI}) \text { from diagnosis }\end{array}$ & $1.27(1.20-1.36)$ & $1.51(1.40-1.62)$ & $0.93(0.80-1.05)$ & $<0.0001^{\mathrm{b}, \mathrm{c}}$ \\
\hline
\end{tabular}

Abbreviation: $\mathrm{Cl}=$ confidence interval.

The figures are from a population sample of patients with ALS in Ireland; those who do not attend clinic are older, have poorer prognosis, and are more likely to have bulbar-onset ALS compared to those who attend clinic. This illustrates the importance of population-based case ascertainment for the study of neurodegenerative diseases.

a Student $t$ test. Others tests are Fisher exact test.

b Significant.

${ }^{\mathrm{c}}$ Mantel-Haenszel rate ratio $=0.657$ for clinic attenders vs nonattenders

prognosis, and introduces bias in the analysis of predictors of prognosis. In addition, although arguably the best possible design for common diseases, large health study cohorts remain at risk from drawing from a narrow socially or geographically defined target population. A restricted definition of the target population undermines one of the key purposes of registers and extreme residential segregation by social factors, as is common in the United States, ${ }^{38}$ can exacerbate this challenge. Solutions to this problem could be to link data from multiple sites, although this would entail substantial challenges in both logistics and funding, or to combine data from different cohort studies, ${ }^{39}$ although this would not resolve the inherent biases within each individual cohort study.

ESSENTIAL CONSIDERATIONS FOR NEW REGISTERS Core content. Population-based registers in neurodegenerative diseases recognize the importance of defining in advance and collecting core content for all cases that fulfil the diagnostic criteria for ascertainment. ${ }^{1,2}$ Standardization permits accurate comparative analyses of different geographic populations and data pooling to enhance statistical power. The NIH/National Institute of Neurological Disorders and Stroke in the United States has recently defined detailed common data elements for a range of neurologic diseases, ${ }^{1}$ and the European Platform for Rare Disease Registries in Europe has proposed a more general set of common data elements for use in rare disease registries. ${ }^{40} \mathrm{~A}$ requirement for excessive detail increases participant burden and the likelihood of missing data, and for this reason extensive clinical data (such as would be required for care pathways) are not suited to registers. Such data are best generated as additional nested studies associated with but not part of the core functioning of a prospective longitudinal register (table 2).

Recognizing ascertainment bias. The principal advantage of the population-based register is the minimization of sources of bias (table 3). Although elimination of all forms of ascertainment bias is not possible in the design and establishment of any new register, a recognition of the likely sources of bias is important. Factors that can assist the reduction of ascertainment biases 
Table 2 Essential design aspects for population-based registers

Register variables should be selected carefully and a core content paradigm should be agreed upon in advance.

Core content should include clearly defined case definitions and these should be applied within a framework of specifically stated inclusion and exclusion criteria.

Capture methodology should be clearly defined in advance.

Case identification pathways should be agreed upon in advance and should include multiple sources for identification of incident cases.

Core content should facilitate international collaborative efforts or national merger of data in large countries using multiple registers to cover different regions is advisable for rare diseases.

Identification of data manager and data controller and clear definition of governance structures is necessary.

Compliance with local data protection legislation and ethica constraints is required.

include clearly defined and rigorously applied geographic boundaries, ${ }^{7,41}$ stability of the population within a designated capture region, and recognition of migration patterns. Prospective design ${ }^{42}$ and longitudinal follow-up with the intention of complete ascertainment of all affected individuals can reduce known bias, as does a stable population structure with limited mobility, which minimizes the risk of bias associated with loss of follow-up.

As noted, no single data source is likely to identify all cases in population-based registers, and multiple sources of identification can improve reliability of ascertainment and ensure that the range and extent of disease heterogeneity is represented.7,42 The use of capture recapture analysis s,15,18,19 $^{6}$ based on 2 or more independent source datasets permits evaluation of the degree of completeness of ascertainment, so that the population burden of cases can be estimated even if no source is complete.

Effective case identification strategies include referrals through networks of clinical professionals, death certification, and face-to-face or telephonebased interviews. Pitfalls that introduce bias include the use of single sources of ascertainment. These include collection of patients exclusively through specialist clinics as noted (tables 1 and 3), self-reporting of patients through online portals, or exclusive reliance on codes from billing data, which are subject to selection bias and diagnostic error. ${ }^{3,43,44}$ Death certification may also overestimate or underestimate disease incidence because of inaccurate recording and coding. ${ }^{45,46}$

Bias of diagnostic uncertainty. Population-based registers require a high level of vigilance to exclude erroneous diagnoses and mimic syndromes in both rare and common diseases. ${ }^{47-49}$

Diagnostic certainty is problematic for both rare and common neurodegenerative diseases for different reasons. Rare diseases may not be recognized outside of specialist centers. Conversely, certainty of diagnosis of common diseases (e.g., dementias) can be limited by the absence of strict diagnostic criteria, and logistics surrounding the acquisition of sufficiently detailed clinical information to ensure clarity for inclusion purposes. This is a particular problem in the ascertainment of cognitive impairment that presages dementia. While memory clinics advocate regular screening, such an initiative is not practical within a population-based setting, and also risks the generation of false-positive diagnoses. ${ }^{28,29}$

Table 3 Common sources of bias and their resolution Bias source
Ascertainment from sources
other than population

Prevalent cases only or mixed incident and prevalent cases recruited

Diagnostic certainty

Startup bias

Information creep

Period and cohort effects

\section{Bias implication}

Incomplete data, very young, very old, and very severe and very mild cases likely to be excluded; economically disadvantaged/ minorities excluded

Milder cases, incomplete clinical phenotypes, and longer natural history

Misdiagnoses and mimic syndromes included

Mixture of incident and prevalent cases included; clinical phenotype and natural history data skewed in favor of milder cases with better survival

Subtle changes in inclusion criteria; differences in clinical phenotype between historical cases and those recently enrolled

Reasons for true differences between different cohorts misattributed

\section{Resolution}

Multiple sources with capture recapture

Longitudinal incident-based inclusion

Quality control, review of source documentation, autopsy reports; excluding the most recent 1-2 years of data capture (or longer if the condition has long lag from initia diagnosis to inclusion on the register)

Recognition, employment of careful statistica analyses of data collected in the first 3-5 years to account for startup bias

Awareness, rigorous use of core data elements; addition of new fields with additional data; correction for missing data from earlier cohorts

Awareness; mathematical correction where possible; rigorous attention and correction for missing data 
Some neurodegenerations have low clinicopathologic correlation, ${ }^{50-52}$ and a recent meta-analysis on diagnosis of PD showed that 1 out of 5 diagnoses of is wrong. ${ }^{53}$ The low clinicopathologic concordance is not intrinsic to register data, but reflects real ambiguity and controversy in the clinical diagnoses, the frequent overlap between different types of neurodegeneration (e.g., $\mathrm{AD}$, vascular dementia, and Lewy body dementia ${ }^{50}$ ), and imperfect correspondence between clinical symptoms and neuropathologic bases of disease in some instances. ${ }^{53}$ Moreover, diagnoses in neurodegeneration may shift with changing symptoms during the course of the disease, and some differential diagnoses (e.g., corticobasal degeneration vs progressive supranuclear palsy) can only be made after specific symptoms develop, or even at the time of autopsy. ${ }^{52}$ These limitations, although difficult to address at the time of ascertainment, should be anticipated in the design of population-based neurodegenerative disease registers by incorporating ongoing collection of clinical data at regular intervals and by regular quality control assessment by review of source documents, correlation with death certificates, and where possible, autopsy reports.

Bias of including mixed incident and prevalent cohorts. Inclusion of both prevalent and incident cases in prospective disease registers introduces bias. Collection of incident cases generally facilitates the characterization of phenotype throughout the disease course, including variability in progression when ascertained longitudinally over an extended period of time. ${ }^{42,54}$ Conversely, inclusion of prevalent cases can bias characterization of the natural history of disease because prevalent cases will on average overrepresent more slowly progressing and less severe cases. ${ }^{54,55}$ This bias is most likely to occur when patients are ascertained from clinic cohorts, which generally cater for a larger proportion of prevalent rather than incident cases. ${ }^{6,26,54-57}$ Clinical trial cohorts such as the Pooled Resource Open-Access ALS Clinical Trials (PRO-ACT) database are also subject to this type of bias. ${ }^{58}$ Population-based registers of incident cases that have been in operation for long periods of time catalogue all incident and prevalent cases and effectively eliminate this source of bias.

Startup bias. Data captured in the early years of a register are unlikely to be of the same quality as subsequently captured data, as there is generally a bias towards enrollment of prevalent rather than incident cases. Also, most registers miss some cases in the early years. Such issues are inevitable in the initiation years of any disease register regardless of how well the methodology of capture of cases is planned. This creates a substantial bias that is rarely recognized, but that must be accounted for in later analyses. As the register develops, the multisource case ascertainment mechanism becomes more streamlined, data are collected prospectively, and the target population becomes aware of the register, reducing rates of missing values. Therefore, startup bias must be considered in interrogating registers of recent origin, especially when evaluating time to event outcomes (e.g., with survival analysis). ${ }^{59}$ This bias also applies to analysis of data from recent case ascertainment in longstanding registers, as there is usually a time lag between case ascertainment and quality control methods that ensure diagnostic accuracy. To minimize these types of bias, analyses should be conducted by excluding data acquired at the beginning of the register, and within a specified period (often the previous year) of recent case ascertainment.

Changes of disease definition. The changing understanding of different clinical diagnoses and their interrelationships presents challenges for registers. For example, there is now a recognition that the clinical condition commonly classified as dementia of the Alzheimer type is heterogeneous, with contributing vascular and inflammatory factors, and the value of detailed phenotype characterization is accordingly increasingly recognized. Similarly, ALS was traditionally considered to be a neurodegenerative disorder confined to the motor system. However, it is now recognized that cognitive and behavioral changes are intrinsic features of the condition. ${ }^{37,60, \mathrm{e} 1}$ The overlap between ALS and FTD has been further recognized following the discovery of the C9orf72 hexanucleotide repeat expansion. ${ }^{\mathrm{e} 2, \mathrm{e} 3}$ Inclusion of cognitive and genetic status is now recognized as an important core element of ALS registers, necessitating adjustment of the existing core datasets, and effectively leading to a large volume of missing data for patients captured by ALS registers in the 1990s and early 2000s.

For these reasons, comparative analyses of register data collected over long periods of time can be problematic, both from the perspective of missing data and in relation to the effect that this new information has on the process of case ascertainment and inferences regarding risk. Moreover, although successful population-based registers may boast complete ascertainment, it is likely that the increased recognition of both comorbidities and subphenotypes can lead to subtle shifts in the types of patients who are included. ${ }^{\mathrm{e}-\mathrm{e} 6}$

As is the case with diagnostic certainty, there is no mechanism to eliminate this type of bias. However, it is important to be aware that it exists, and that comparative analyses of data across time intervals will be subject to the bias of information creep.

In addition to the bias of evolving information and subtle changes in diagnostic categorizations, period 
and cohort effects should also be recognized during comparative analyses across different time periods of an individual register, and across registers from different regions and of different durations. It cannot be assumed that populations ascertained by registers at different time intervals are the same, and these effects should be taken into account when performing comparative analyses. For these reasons, the use of historical controls in the evaluation of interventions introduces a bias that cannot be evaluated.

CHALLENGES IN MANAGING LONGITUDINAL REGISTERS Logistics and infrastructure. Registers are extremely vulnerable to interruptions in funding. Disease registers are cost- and labor-intensive, and (unlike EHRs) require active management to ensure uniformity of ascertainment and ongoing quality control. The costs of establishing and maintaining a register will depend on (1) the size of the study population, (2) the type of disease (e.g., common or rare), and (3) the nature of the health service within which the register operates. In all cases, sustainable funding is challenging in the absence of a specific government/ funding agency policy commitment, such as has occurred in the United States with the inception of the US ALS register, ${ }^{\mathrm{e}, \mathrm{e} 8}$ and in Denmark, where the is a strong historical tradition of supporting high-quality longitudinal registers with interlocking datasets that are open to interrogation. ${ }^{\mathrm{e}}$ With these exceptions, disease registers continue to be viewed by many funding agencies as infrastructural resources rather than essential research instruments, and are excluded from funding as they do not of themselves generate research outputs. Indeed, in a recent survey of European rare disease registers, 23\% of 202 registers were established with no specific source of funding. ${ }^{\text {e10 }}$ Unstable funding streams devalue the original investments in establishing a register because the data become more valid, comprehensive, and reliable over years as the register matures.

Ethics and data protection legislation. The sustainability of registers in the longer term is challenged by ongoing limitations on the types of data that disease registers are likely to be permitted to record. Data protection legislation has been strengthened within the last 20 years, and inclusion of data regarding living patients without their expressed informed consent in the absence of specific legislation is now in breach of data protection laws in Europe. This has the potential to introduce bias.

These problems could be addressed by legislation providing a derogation for population-based registers from the full strictures of data protection legislation as in the case of the Nebraska Parkinson's Disease Register, ${ }^{15}$ while maintaining strict control in the types of access permitted. This requires an understanding and recognition by the public of the important potential societal benefits of population-based epidemiologic research, and in particular the potential public health benefit of identifying and communicating deidentified data regarding regional variations in disease incidence, prevalence, and survival. This benefit, which is implicit in the case of notifiable infectious and communicable diseases, is juxtaposed with the right to privacy of the individual. While some countries created registers that are now core research tools (e.g., the US ALS registry, the Danish registers, and cancer registers in many countries), there remains a limited recognition within Europe of the public health and societal benefits of such an approach for most noncancer and noncommunicable disease from the dual perspectives of research and public health/policy. The issues of privacy and data protection must be clearly addressed in registers and data should be codified to exclude personal identification. Clearly enunciated protocols should be in place to determine who can access the data, and the circumstances under which this access is permitted. Nevertheless, the goals of fostering access by qualified researchers and preventing reidentification are sometimes in competition. Recent efforts to enhance data-sharing throughout the health research community have fostered innovations in data security, and investment in adopting these protocols and updating as new technologies become available is important to ensure the confidentiality of participants while maximizing research applications.

DISCUSSION Acknowledgment, recognition, and rectification of the inherent biases within populationbased datasets is necessary for accurate demographic and deep phenotypic characterization of neurodegenerative diseases. However, some biases within registers can never be fully avoided. Notwithstanding, appropriate interrogation of register data can build our understanding of clinical and biological heterogeneity of neurodegenerative diseases within and across different populations. Well-designed population-based registers are essential to the provision of accurate epidemiologic and phenotypic characterization that will drive understanding of the heterogeneous disease mechanisms underpinning rare and common neurodegenerative diseases.

\section{AUTHOR CONTRIBUTIONS}

O.H. conceived the study and contributed to the writing of first and subsequent drafts. J.P.K.R. wrote the first draft, performed the literature search, and prepared tables. K.T. prepared the figure and contributed to the writing of the first draft. C.B., M.M.G., and G.L. provided expert review of the first draft and contributed to subsequent drafts. All authors approved the final manuscript.

\section{STUDY FUNDING}

J.P.K.R., K.T., and O.H. are funded by the Health Research Board (Ireland). 


\section{DISCLOSURE}

J. Rooney was funded by the Health Research Programme Clinical Fellowship Programme. C. Brayne reports no disclosures relevant to the manuscript. K. Tobin is funded by the HRB Interdisciplinary Capacity Enhancement Fellowship Programme. She reports no conflicts of interest. G. Logroscino serves as associate editor for the Neuroepidemiology Journal, Karger. He received founding from Ministry of Health. Ministry of University and Research, regione Puglia Special program for research. M. Glymour is funded by the NIH, the Robert Wood Johnson Foundation, and the University of California, San Francisco. She reports no conflicts of interest. O. Hardiman is funded by the Health Research Board Clinician Scientist Programme and Science Foundation Ireland (15/SPP/3244 and 16/ERCD/3854). Prof. Hardiman has received speaking honoraria from Novartis, Biogen Idec, Sanofi Aventis, and MerckSerono. She has been a member of advisory panels for Biogen Idec, Allergen, Ono Pharmaceuticals, Novartis, Cytokinetics, and Sanofi Aventis. She serves as Editor-in-Chief of Amyotrophic Lateral Sclerosis and Frontotemporal Dementia. Go to Neurology.org for full disclosures.

Received November 9, 2016. Accepted in final form March 24, 2017.

\section{REFERENCES}

1. NINDS/NIH. NINDS Common Data Elements. 2015. Available at: commondataelements.ninds.nih. gov/\#page $=$ Default. Accessed November 18, 2015.

2. ENCALS. ALS Core Clinical Dataset. 2014. Available at: encals.eu/public/media/ac8bacd302965623601ddfe4229c 4aadcc370922/6xg7/pdf/17a00a103f602f2837d968fd03 8df3ca17ae0c76.pdf. Accessed November 18, 2015.

3. Vasta R, Boumédiene F, Couratier P, et al. Validity of medico-administrative data related to amyotrophic lateral sclerosis in France: a population-based study. Amyotroph Lateral Scler Frontotemporal Degener 2017;18:24-31.

4. Religa D, Fereshtehnejad SM, Cermakova P, et al. SveDem, the Swedish dementia registry: a tool for improving the quality of diagnostics, treatment and care of dementia patients in clinical practice. PLoS One 2015;10:e0116538.

5. Anthony S, Pradier C, Chevrier R, Festraëts J, Tifratene K, Robert P. The French National Alzheimer database: a fast growing database for researchers and clinicians. Dement Geriatr Cogn Disord 2014;38:271-280.

6. Huisman MHB, De Jong SW, Van Doormaal PTC, et al. Population based epidemiology of amyotrophic lateral sclerosis using capture-recapture methodology. J Neurol Neurosurg Psychiatry 2011;82:1165-1170.

7. Beghi E, Logroscino G, Chiò A, et al. The epidemiology of ALS and the role of population-based registries. Biochim Biophys Acta 2006;1762:1150-1157.

8. Traynor BJ, Codd MB, Corr B, Forde C, Frost E, Hardiman O. Incidence and prevalence of ALS in Ireland, 1995-1997: a population-based study. Neurology 1999;52:504.

9. Rosso SM, Kaat LD, Baks T, et al. Frontotemporal dementia in The Netherlands: patient characteristics and prevalence estimates from a population-based study. Brain 2003;126:2016-2022.

10. Knopman DS, Roberts RO. Estimating the number of persons with frontotemporal lobar degeneration in the US population. J Mol Neurosci 2011;45:330-335.

11. Onyike CU, Diehl-Schmid J. The epidemiology of frontotemporal dementia. Int Rev Psychiatry 2013;25:130-137.

12. Forbes RB, Colville $S$, Swingler RJ. The epidemiology of amyotrophic lateral sclerosis (ALS/MND) in people aged 80 or over. Age Ageing 2004;33:131-134.
13. Rooney J, Vajda A, Heverin M, et al. Spatial cluster analysis of population amyotrophic lateral sclerosis risk in Ireland. Neurology 2015;84:1537-1544.

14. Pupillo E, Messina P, Logroscino G, Beghi E. Long-term survival in amyotrophic lateral sclerosis: a populationbased study. Ann Neurol 2014;75:287-297.

15. Strickland D, Bertoni JM. Parkinson's prevalence estimated by a state registry. Mov Disord 2004;19:318-323.

16. Tolppanen AM, Lavikainen P, Solomon A, Kivipelto M, Soininen H, Hartikainen S. Incidence of stroke in people with Alzheimer disease: a national register-based approach. Neurology 2013;80:353-358.

17. Nebraska DHHS. Nebraska Parkinson's Disease Registry. Available at: dhhs.ne.gov/publichealth/Pages/ced_parkinsons_ index.aspx. Accessed December 8, 2015.

18. Hook EB, Regal RR. Effect of variation in probability of ascertainment by sources ("variable catchability") upon "capture-recapture" estimates of prevalence. Am J Epidemiol 1993;137:1148-1166.

19. Chao A. Capture-recapture for human populations. In: Wiley StatsRef: Statistics Reference Online. Chichester: John Wiley \& Sons; 2015:1-16.

20. Bhidayasiri R, Wannachai N, Limpabandhu S, et al. A national registry to determine the distribution and prevalence of Parkinson's disease in Thailand: implications of urbanization and pesticides as risk factors for Parkinson's disease. Neuroepidemiology 2011;37:222-230.

21. Frandsen R, Baandrup L, Kjellberg J, Ibsen R, Jennum $P$. Increased all-cause mortality with psychotropic medication in Parkinson's disease and controls: a national register-based study. Parkinsonism Relat Disord 2014; 20:1124-1128.

22. Kosteniuk JG, Morgan DG, O'Connell ME, et al. Incidence and prevalence of dementia in linked administrative health data in Saskatchewan, Canada: a retrospective cohort study. BMC Geriatr 2015;15:73.

23. Rocca WA, Petersen RC, Knopman DS, Hebert LE. Trends in the incidence and prevalence of Alzheimer's disease, dementia and cognitive impairment in the United States. Alzheimers Dement 2012;7:80-93.

24. Tsai CP, Tzu-Chi Lee C. Climatic factors associated with amyotrophic lateral sclerosis: a spatial analysis from Taiwan. Geospat Health 2013;8:45-52.

25. Solomon A, Ngandu T, Soininen H, Hallikainen MM, Kivipelto M, Laatikainen T. Validity of dementia and Alzheimer's disease diagnoses in Finnish national registers. Alzheimers Dement 2014;10:303-309.

26. Brayne C, Davis D. Making Alzheimer's and dementia research fit for populations. Lancet 2012;380:1441-1443.

27. Aragones JM, Altimiras J, Roura-Poch P, et al. Amyotrophic lateral sclerosis: a higher than expected incidence in people over 80 years of age. Amyotroph Lateral Scler Frontotemporal Degener 2016;8421:1-6.

28. Petersen RC. Mild cognitive impairment as a clinical entity and treatment target. Arch Neurol 2004;62:11601163.

29. Matthews FE, Stephan BCM, McKeith IG, Bond J, Brayne C. Two-year progression from mild cognitive impairment to dementia: to what extent do different definitions agree? J Am Geriatr Soc 2008;56:1424-1433.

30. Schoenmaker N, Van Gool WA. The age gap between patients in clinical studies and in the general population: a pitfall for dementia research. Lancet Neurol 2004;3: 627-630. 
31. Jak AJ, Preis SR, Beiser AS, et al. Neuropsychological criteria for mild cognitive impairment and dementia risk in the Framingham Heart Study. J Int Neuropsychol Soc 2016;22:937-943.

32. Lagergren M, Fratiglioni L, Hallberg IR, et al. A longitudinal study integrating population, care and social services data: The Swedish National study on Aging and Care (SNAC). Aging Clin Exp Res 2004;16:158-168.

33. Hofman A, Brusselle GGO, Murad SD, et al. The Rotterdam Study: 2016 objectives and design update. Eur J Epidemiol 2015;30:661-708.

34. Erasmus. The Rotterdam Study. Available at: erasmusmc. $\mathrm{nl} /$ epi/research/The-Rotterdam-Study/?lang=en. Accessed November 10, 2015.

35. de Bruijn RFAG, Bos MJ, Portegies MLP, et al. The potential for prevention of dementia across two decades: the prospective, population-based Rotterdam Study. BMC Med 2015;13:132.

36. Glymour MM, Chêne G, Tzourio C, Dufouil C. Brain MRI markers and dropout in a longitudinal study of $\operatorname{cog}$ nitive aging: the Three-City Dijon Study. Neurology 2012;79:1340-1348.

37. Elamin M, Bede P, Byrne S, et al. Cognitive changes predict functional decline in ALS: a population-based longitudinal study. Neurology 2013;80:1590-1597.

38. Fry R, Taylor P. The Rise of Residential Segregation by Income. Washington, DC: Pew Research Center; 2012.

39. Harrison SL, de Craen AJM, Kerse N, et al. Predicting risk of cognitive decline in very old adults using three models: the Framingham stroke risk profile; the cardiovascular risk factors, aging, and dementia model; and oxiinflammatory biomarkers. J Am Geriatr Soc 2017;65: 381-389.

40. Taruscio D, Mollo E, Gainotti S, Posada de la Paz M, Bianchi F, Vittozzi L. The EPIRARE proposal of a set of indicators and common data elements for the European platform for rare disease registration. Arch Public Health 2014;72:35.

41. Logroscino G, Traynor BJ, Hardiman O, et al. Incidence of amyotrophic lateral sclerosis in Europe. J Neurol Neurosurg Psychiatry 2010;81:385-390.

42. Chiò A, Logroscino G, Traynor BJ, et al. Global epidemiology of amyotrophic lateral sclerosis: a systematic review of the published literature. Neuroepidemiology 2013;41: 118-130.

43. Klovning A, Sandvik H, Hunskaar S. Web-based survey attracted age-biased sample with more severe illness than paper-based survey. J Clin Epidemiol 2009;62:1068-1074.

44. Manuel DG, Rosella LC, Stukel TA. Importance of accurately identifying disease in studies using electronic health records. BMJ 2010;341:c4226.
45. Yeo L, Lynch C, Hardiman O. Validating populationbased registers for ALS: how accurate is death certification? J Neurol 2010;257:1235-1239.

46. Perera G, Stewart R, Higginson IJ, Sleeman KE. Reporting of clinically diagnosed dementia on death certificates: retrospective cohort study. Age Ageing 2016;45:668-673.

47. Traynor BJ, Codd MB, Corr B, Forde C, Frost E, Hardiman $\mathrm{O}$. Amyotrophic lateral sclerosis mimic syndromes. Arch Neurol 2000;57:109.

48. Possin KL, Kaufer DI. Parkinsonian dementias. Continuum 2010;16:57-79.

49. Sáez-Fonseca JA, Lee L, Walker Z. Long-term outcome of depressive pseudodementia in the elderly. J Affect Disord 2007;101:123-129.

50. Shim YS, Roe CM, Buckles VD, Morris JC. Clinicopathologic study of Alzheimer's disease: Alzheimer mimics. J Alzheimers Dis 2013;35:799-811.

51. Adler CH, Beach TG, Hentz JG, et al. Low clinical diagnostic accuracy of early vs advanced Parkinson disease: clinicopathologic study. Neurology 2014;83:406-412.

52. Horvath J, Burkhard PR, Bouras C, Kövari E. Etiologies of Parkinsonism in a century-long autopsy-based cohort. Brain Pathol 2013;23:28-33.

53. Rizzo G, Copetti M, Arcuti S, Martino D, Fontana A, Logroscino G. Accuracy of clinical diagnosis of Parkinson disease: a systematic review and meta-analysis. Neurology 2016;86:566-576.

54. Toole OO, Traynor BJ, Brennan P, et al. Epidemiology and clinical features of amyotrophic lateral sclerosis in Ireland between 1995 and 2004. J Neurol Neurosurg Psychiatry 2008;79:30-32.

55. Lee JRJ, Annegers JF, Appel SH. Prognosis of amyotrophic lateral sclerosis and the effect of referral selection. J Neurol Sci 1995;132:207-215.

56. Taruscio D, Vittozzi L, Choquet R, et al. National registries of rare diseases in Europe: an overview of the current situation and experiences. Public Health Genomics 2015; 18:20-25.

57. Teschke K, Marion SA, Tsui JKC, Shen H, Rugbjerg K, Harris MA. Parkinson's disease and occupation: differences in associations by case identification method suggest referral bias. Am J Ind Med 2014;57:163-171.

58. Berry J, Shui A, Zach N, Schoenfeld D. The PRO-ACT database: design, initial analyses, and predictive features. Neurology 2014;83:1719-1725.

59. Rooney J, Byrne S, Heverin M, et al. Survival analysis of Irish amyotrophic lateral sclerosis patients diagnosed from 1995-2010. PLoS One 2013;8:e74733.

60. Elamin M, Phukan J, Bede P, et al. Executive dysfunction is a negative prognostic indicator in patients with ALS without dementia. Neurology 2011;76:1263-1269. 